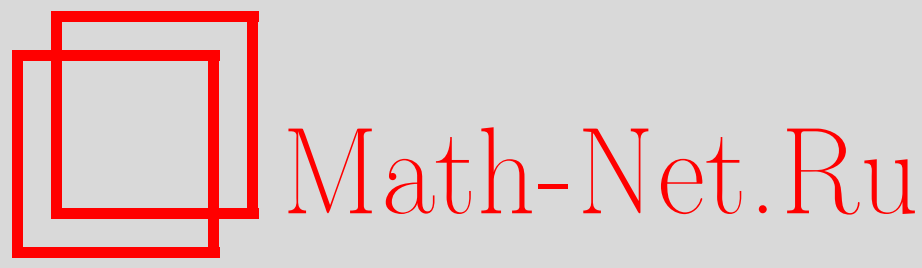

Н. М. Хатамов, Новые классы основных состояний для модели Поттса с рассеянными конкурирующими взаимодействиями на дереве Кэли, ТМФ, 2014, том 180, номер 1, 86-93

DOI: https://doi.org/10.4213/tmf8637

Использование Общероссийского математического портала Math-Net.Ru подразумевает, что вы прочитали и согласны с пользовательским соглашением http: //www.mathnet.ru/rus/agreement

Параметры загрузки:

IP : 54.81 .137 .203

26 апреля 2023 г., 17:39:19

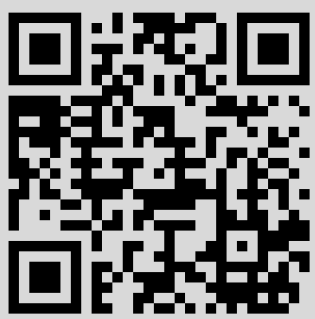




\title{
ФИЗИКА
}

Том 180, № 1

июль, 2014

(C) 2014 г.

\author{
Н. М. Хатамов*
}

\section{НОВЫЕ КЛАССЫ ОСНОВНЫХ СОСТОЯНИЙ ДЛЯ МОДЕЛИ ПОТТСА С РАССЕЯННЫМИ КОНКУРИРУЮЩИМИ ВЗАИМОДЕЙСТВИЯМИ НА ДЕРЕВЕ КЭЛИ}

Рассматривается модель Поттса с рассеянными конкурирующими взаимодействиями на дереве Кэли. Для этой модели изучается область основных состояний.

Ключевые слова: дерево Кэли, модель Поттса, основные состояния.

DOI: $10.4213 / \operatorname{tmf} 8637$

Основные состояния для моделей статистической физики на дереве Кэли изучены в работах [1]-[4]. В работе [5] исследована структура основных состояний для модели Изинга с взаимодействиями на три шага на решетке $\mathbb{Z}^{3}$. В настоящей работе рассматривается модель Поттса с рассеянными конкурирующими взаимодействиями на дереве Кэли и для этой модели изучается структура основных состояний.

Дерево Кэли (или решетка Бете в другой терминологии) $\Gamma^{k}$ порядка $k \geqslant 1$ представляет собой бесконечное дерево, т. е. граф без циклов, из каждой вершины которого выходит ровно $k+1$ ребер [1]-[4]. Пусть $\Gamma^{k}=(V, L, i)$, где $V$ - множество вершин $\Gamma^{k}, L$ - его множество ребер, $i$ - функция инцидентности, сопоставляющая каждому ребру $l \in L$ его концевые точки $x, y \in V$. Если $i(l)=\{x, y\}$, то $x$ и $y$ называются ближайшими соседями, и вводится обозначение $l=\langle x, y\rangle$. Расстояние $d(x, y)$ для любых $x, y \in V$ на дереве Кэли определяется формулой

$$
d(x, y)=\min \left\{d \mid \exists x=x_{0}, x_{1}, \ldots, x_{d}=y \in V \quad \text { такой, что } \quad\left\langle x_{0}, x_{1}\right\rangle, \ldots,\left\langle x_{d-1}, x_{d}\right\rangle\right\} .
$$

Известно, что существует взаимно однозначное соответствие между множеством $V$ вершин дерева Кэли порядка $k \geqslant 1$ и группой $G_{k}$ свободных произведений $k+1$ циклической группы второго порядка с образующими $a_{1}, a_{2}, \ldots, a_{k+1}$ соответственно.

Рассмотрим модель, в которой спин принимает значения из множества

$$
\Phi=\{1,2, \ldots, q\}, \quad q \geqslant 2 .
$$

*Наманганский государственный университет, Наманган, Узбекистан. E-mail: nxatamov@mail.ru 
Тогда конфигурация $\sigma$ на $V$ определяется как функция $x \in V \rightarrow \sigma(x) \in \Phi$; множество всех конфигураций совпадает с $\Omega=\Phi^{V}$.

Предположим, что на множестве $\Omega$ действует группа пространственных переходов. Определим $F_{k}$-периодическую конфигурацию $\sigma(x)$, которая является инвариантной относительно подгруппы $F_{k} \subset G_{k}$ конечного индекса, т. е. $\sigma(y x)=\sigma(x)$ для любых $x \in G_{k}, y \in F_{k}$. Для данной периодической конфигурации индекс подгруппы называется периодом конфигурации. Конфигурация, которая является инвариантной относительно всех переходов, называется трансляиионно-инвариантной.

Пусть $\left\langle x^{0}, x^{1}\right\rangle=l \in L-$ фиксированное ребро (см. [6]). Рассмотрим два полубесконечных дерева $\Gamma_{0}^{k}$ и $\Gamma_{1}^{k}$, где $\Gamma_{0}^{k}$ - дерево, растущее из вершины $x^{0} \in V$ "вверх", проходящее через $x^{1}$, а $\Gamma_{1}^{k}$ - дерево, растущее из вершины $x^{1} \in V$ "вниз", проходящее через $x^{0}$.

На деревьях $\Gamma_{0}^{k}$ и $\Gamma_{1}^{k}$ можно ввести частичное упорядочение, сказав, что $y>x$, если $x=x_{0}, x_{1}, x_{2}, \ldots, x_{n}=y$ из $x$ в $y$, "идущий вверх" для $\Gamma_{0}^{k}$, а для $\Gamma_{1}^{k}$ "идущий вниз", т. е. $d\left(x_{k}, x^{i}\right)=d\left(x_{k-1}, x^{i}\right)+1, k=1, \ldots, n, i=0,1$.

Вершина $y$ называется прямым потомком $x$, если $y>x$ и $x, y$ являются ближайшими соседями.

Множество прямых потомков точки $x$ на дереве $\Gamma_{0}^{k}$ лежит сверху, а на дереве $\Gamma_{1}^{k}-$ снизу от точки $x$.

Пусть $\Gamma_{0}^{k}=\left(V^{0}, L^{0}\right)$ и $\Gamma_{1}^{k}=\left(V^{1}, L^{1}\right)$, т. е. $V^{i}$ - множество вершин $\Gamma_{i}^{k}, L^{i}$ - множество его ребер, $i=0,1$. Понятно, что $V^{0} \cup V^{1}=V, L^{0} \cup L^{1}=L$.

Если точки $x, y \in V$ являются прямыми потомками некоторой вершины, то точки $x, y \in V$ называются вершинами, лежащими на одном “этаже”, и обозначаются \rangle $x, y\langle$. Ясно, что $d(x, y)=2$.

Если точки $x, y \in V$ такие, что $d(x, y)=2$ и только одна из них является прямым потомком некоторой вершины, то $x, y \in V$ называются вершинами, лежащими на разных "этажсах", и обозначаются $\rangle\rangle x, y\langle\langle$.

Гамильтониан модели Поттса с рассеянными конкурирующими взаимодействиями имеет вид

$$
H(\sigma)=J_{1} \sum_{\langle x, y\rangle ; x, y \in V} \delta_{\sigma(x) \sigma(y)}+J_{2} \sum_{\rangle x, y\langle; x, y \in V} \delta_{\sigma(x) \sigma(y)}+J_{3} \sum_{\lambda\rangle x, y\langle\langle; x, y \in V} \delta_{\sigma(x) \sigma(y)},
$$

где $J_{1}, J_{2}, J_{3} \in \mathbb{R}$,

$$
\delta_{u v}= \begin{cases}1, & u=v \\ 0, & u \neq v\end{cases}
$$

Для пары конфигураций $\sigma$ и $\varphi$, совпадающих почти всюду, т. е. всюду, за исключением конечного числа точек, мы рассмотрим относительный гамильтониан $H(\sigma, \varphi)-$ различие между энергиями конфигураций $\sigma, \varphi$, т. е.

$$
\begin{aligned}
H(\sigma, \varphi)=J_{1} & \sum_{\langle x, y\rangle ; x, y \in V}\left(\delta_{\sigma(x) \sigma(y)}-\delta_{\varphi(x) \varphi(y)}\right)+J_{2} \sum_{>x, y\langle; x, y \in V}\left(\delta_{\sigma(x) \sigma(y)}-\delta_{\varphi(x) \varphi(y)}\right)+ \\
& +J_{3} \sum_{>\rangle x, y\langle\langle; x, y \in V}\left(\delta_{\sigma(x) \sigma(y)}-\delta_{\varphi(x) \varphi(y)}\right)
\end{aligned}
$$

где $J=\left(J_{1}, J_{2}, J_{3}\right) \in \mathbb{R}^{3}$ - произвольный фиксированный параметр. 
Пусть $M$ - множество единичных шаров с вершинами в $V$. Будем называть сужение конфигурации $\sigma$ на шаре $b \in M$ ограниченной конфигурацией $\sigma_{b}$. Определим энергию конфигурации $\sigma_{b}$ на шаре $b$ следующим образом:

$$
\begin{gathered}
U\left(\sigma_{b}\right) \equiv U\left(\sigma_{b}, J\right)=\frac{1}{2} J_{1} \sum_{\langle x, y\rangle ; x, y \in b} \delta_{\sigma(x) \sigma(y)}+J_{2} \sum_{\gamma x, y\langle; x, y \in b} \delta_{\sigma(x) \sigma(y)}+ \\
+J_{3} \sum_{>\rangle x, y\langle\langle; x, y \in b} \delta_{\sigma(x) \sigma(y)},
\end{gathered}
$$

где $J=\left(J_{1}, J_{2}, J_{3}\right) \in \mathbb{R}^{3}$.

Из этого определения для относительного гамильтониана можно получить следующую лемму.

ЛЕмма 1. Относительный гамильтониан (2) имеет вид

$$
H(\sigma, \varphi)=\sum_{b \in M}\left(U\left(\sigma_{b}\right)-U\left(\varphi_{b}\right)\right)
$$

Рассмотрим случай $k=2$ и $q=3$. Легко видеть, что $U\left(\sigma_{b}\right) \in\left\{U_{1}, U_{2}, \ldots, U_{9}\right\}$ для любого $\sigma_{b}$, где

$$
\begin{gathered}
U_{1}=\frac{3}{2} J_{1}+2 J_{2}+J_{3}, \quad U_{2}=J_{1}+J_{3}, \quad U_{3}=2 J_{2}+J_{3}, \quad U_{4}=\frac{1}{2} J_{1}+J_{3}, \\
U_{5}=J_{3}, \quad U_{6}=J_{1}+J_{2}, \quad U_{7}=\frac{1}{2} J_{1}+J_{2}, \quad U_{8}=J_{2}, \quad U_{9}=\frac{1}{2} J_{1} .
\end{gathered}
$$

ОПРЕДЕЛЕНИЕ 1. Конфигурация $\varphi$ называется основным состоянием относительного гамильтониана $H$, если $U\left(\varphi_{b}\right)=\min \left\{U_{1}, U_{2}, \ldots, U_{9}\right\}$ для любого $b \in M$.

Обозначим $C_{i}=\left\{\sigma_{b}: U\left(\sigma_{b}\right)=U_{i}\right\}$ и $U_{i}(J)=U\left(\sigma_{b}, J\right)$, если $\sigma_{b} \in C_{i}, i=1, \ldots, 9$.

Tеорема 1. A. Пусть $C$ - один из классов $C_{1}, C_{2} \cup C_{6}, C_{3}, C_{9}, C_{5} \cup C_{8} \cup C_{9}, a \sigma_{b} \in$ $C$ - произвольная ограниченная конфигурация. Тогда существует периодическая конфигурация $\varphi$ (на дереве Кэли) с периодом $p \leqslant 8$ такая, что $\varphi_{b^{\prime}}$ принадлежит $C$ для любого $b^{\prime} \in M u \varphi_{b}=\sigma_{b}$.

Б. Пусть $C$ - один из классов $C_{5}, C_{8}, C_{3} \cup C_{5}, C_{5} \cup C_{8}, a \sigma_{b} \in C$ - произвольная конфигуращия. Тогда существует конфигуращия $\varphi$ (вообще говоря, не являющаяся периодической) такая, что $\varphi_{b^{\prime}} \in C$ для любого $b^{\prime} \in M u \varphi_{b}=\sigma_{b}$.

ДоказАтельство. А. Для произвольного класса $C \in\left\{C_{1}, C_{2} \cup C_{6}, C_{3}, C_{9}, C_{5} \cup\right.$ $\left.C_{8} \cup C_{9}\right\}$ и $\sigma_{b} \in C$ построим конфигурацию $\varphi$ следующим образом: без ограничения общности мы можем в качестве шара $b$ взять шар с центром $e \in G_{2}$ (где $e-$ единичный элемент $\left.G_{2}\right)$, т. е. $b=\left\{e, a_{1}, a_{2}, a_{3}\right\}$.

Рассмотрим несколько случаев.

Случай $C_{1}$. В этом случае мы имеем $\sigma(x)=i, i=1,2,3$, для любого $x \in b$. Таким образом, конфигурация $\varphi$ совпадает с трансляционно-инвариантной, т. е. $\varphi^{(i)}=\left\{\varphi^{(i)}(x)=i\right\}$, где $i=1,2,3$. 
Случай $C_{2} \cup C_{6}$. Рассмотрим $H_{2}=\left\{x \in G_{2}: \omega_{2}(x)\right.$ четно $\}$, где $\omega_{2}(x)$ - число букв $a_{2}$ в слове $x \in G_{2}$. Отметим, что $H_{2}$ - нормальная подгруппа индекса 2 для $G_{2}$ (см. [4]). По построению мы имеем $\sigma_{b}(x)=i$ для любого $x \in b \cap H_{2}$ и $\sigma(x)=j$ для любого $x \in b \cap\left(G_{2} \backslash H_{2}\right)$, где $i, j=1,2,3, i \neq j$.

Продолжим ограниченную конфигурацию $\sigma_{b} \in C_{2} \cup C_{6}$ на целую решетку $\Gamma^{2}$ (которую обозначим $\left.\varphi^{(i j)}\right)$ следующим образом:

$$
\varphi^{(i j)}(x)= \begin{cases}i, & x \in H_{2}, \\ j, & x \in G_{2} \backslash H_{2}\end{cases}
$$

где $i, j=1,2,3, i \neq j$.

Случай $C_{3}$. В этом случае рассмотрим $H_{3}=\left\{x \in G_{2}:|x|\right.$ четно $\}$, где $|x|$ - длина слова $x$. Отметим, что $H_{3}$ также является нормальной подгруппой группы $G_{2}$ индекса 2 (см. [4]). В этом случае конфигурация $\psi^{(i j)}$ может быть определена как

$$
\psi^{(i j)}(x)= \begin{cases}i, & x \in H_{3}, \\ j, & x \in G_{2} \backslash H_{3}\end{cases}
$$

где $i, j=1,2,3, i \neq j$. Таким образом, мы получаем периодическую конфигурацию $\psi^{(i j)}$ с периодом $p=2$ (равным индексу подгруппы); ясно, что $\psi_{b}^{(i j)}=\sigma_{b}$.

Случай $C_{9}$. Пусть $S_{3}$ - группа перестановок третьего порядка. Возьмем следующие $\pi_{0}, \pi_{1}, \pi_{2} \in S_{3}$ :

$$
\pi_{0}=\left(\begin{array}{lll}
1 & 2 & 3 \\
1 & 2 & 3
\end{array}\right), \quad \pi_{1}=\left(\begin{array}{lll}
1 & 2 & 3 \\
2 & 1 & 3
\end{array}\right), \quad \pi_{2}=\left(\begin{array}{lll}
1 & 2 & 3 \\
1 & 3 & 2
\end{array}\right) .
$$

Легко видеть, что $\pi_{0}=\pi_{1}^{2}=\pi_{2}^{2}$. Рассмотрим отображение $u:\left\{a_{1}, a_{2}, a_{3}\right\} \mapsto\left\{\pi_{0}, \pi_{1}, \pi_{2}\right\}$ :

$$
u\left(a_{1}\right)= \begin{cases}\pi_{0}, & i=3 \\ \pi_{i}, & i=1,2\end{cases}
$$

и пусть функция $f: G_{2} \mapsto S_{3}$ определена следующим образом:

$$
f(x)=f\left(a_{i_{1}} a_{i_{2}} \cdots a_{i_{n}}\right)=u\left(a_{i_{1}}\right) \cdots u\left(a_{i_{n}}\right) .
$$

Ясно, что $H_{4}=\left\{x \in G_{2}: f(x)=\pi_{0}\right\}$ - нормальная подгруппа с индексом 6 (см. [4]). Пусть $G_{2} / H_{4}=\left\{\aleph_{0}, \ldots, \aleph_{5}\right\}$ - фактор-группа. В этом случае мы определим конфигурацию $\varphi^{(i j p)}$ следующим образом:

$$
\varphi^{(i j p)}(x)= \begin{cases}i, & x \in \aleph_{0} \cup \aleph_{3}, \\ j, & x \in \aleph_{1} \cup \aleph_{5}, \\ p, & x \in \aleph_{2} \cup \aleph_{4} .\end{cases}
$$

Таким образом, мы получим периодическую конфигурацию $\varphi^{(i j p)}$ с периодом $p=6$, со свойством $\varphi_{b}^{(i j p)}=\sigma_{b}$. 
Случай $C_{5} \cup C_{8} \cup C_{9}$. В этом случае рассмотрим нормальные подгруппы $H_{2}$, $H_{3}$ и $H_{12}=\left\{x \in G_{2}: \omega_{1}(x)+\omega_{2}(x)\right.$ четно $\}$, где $H_{12}$ также является нормальной подгруппой индекса 2 для $G_{2}$ (см. [4]).

Введем следующие обозначения:

$$
\begin{aligned}
& \mathbb{Z}_{0}=H_{12} \cap H_{3} \cap H_{2}, \\
& \mathbb{Z}_{1}=H_{12} \cap H_{3} \cap\left(G_{2} \backslash H_{2}\right), \\
& \mathbb{Z}_{2}=H_{12} \cap\left(G_{2} \backslash H_{3}\right) \cap H_{2}, \\
& \mathbb{Z}_{3}=H_{12} \cap\left(G_{2} \backslash H_{3}\right) \cap\left(G_{2} \backslash H_{2}\right), \\
& \mathbb{Z}_{4}=\left(G_{2} \backslash H_{12}\right) \cap H_{3} \cap H_{2}, \\
& \mathbb{Z}_{5}=\left(G_{2} \backslash H_{12}\right) \cap H_{3} \cap\left(G_{2} \backslash H_{2}\right), \\
& \mathbb{Z}_{6}=\left(G_{2} \backslash H_{12}\right) \cap\left(G_{2} \backslash H_{3}\right) \cap H_{2}, \\
& \mathbb{Z}_{7}=\left(G_{2} \backslash H_{12}\right) \cap\left(G_{2} \backslash H_{3}\right) \cap\left(G_{2} \backslash H_{2}\right) .
\end{aligned}
$$

Известно (см. [4]), что множество $\mathbb{Z}_{0}$ является нормальной подгруппой индекса 8 группы $G_{2}$ и образует $G_{2} / \mathbb{Z}_{0}=\left\{\mathbb{Z}_{0}, \mathbb{Z}_{1}, \mathbb{Z}_{2}, \mathbb{Z}_{3}, \mathbb{Z}_{4}, \mathbb{Z}_{5}, \mathbb{Z}_{6}, \mathbb{Z}_{7}\right\}$ - фактор-группу. В этом случае мы определим конфигурацию $\psi^{(i j p)}$ следующим образом:

$$
\psi^{(i j p)}= \begin{cases}i, & x \in \mathbb{Z}_{0} \cup \mathbb{Z}_{1} \cup \mathbb{Z}_{2} \cup \mathbb{Z}_{3} \\ j, & x \in \mathbb{Z}_{4} \cup \mathbb{Z}_{7} \\ p, & x \in \mathbb{Z}_{5} \cup \mathbb{Z}_{6}\end{cases}
$$

где $i, j, p=1,2,3, i \neq j \neq p$. Таким образом, мы получим периодическую конфигурацию $\psi^{(i j p)}$ с периодом $p=8$ со свойством $\psi_{b}^{(i j p)}=\sigma_{b}$.

Б. Случай $C_{5}$. Для $x \in G_{2}$ введем обозначения $l(x)=l\left(a_{i_{1}} \cdots a_{i_{n}}\right)=i_{1}, r(x)=$ $r\left(a_{i_{1}} \cdots a_{i_{n}}\right)=i_{n}$. В этом случае мы можем определить $\psi_{1}^{(i j p)}(x)$ как

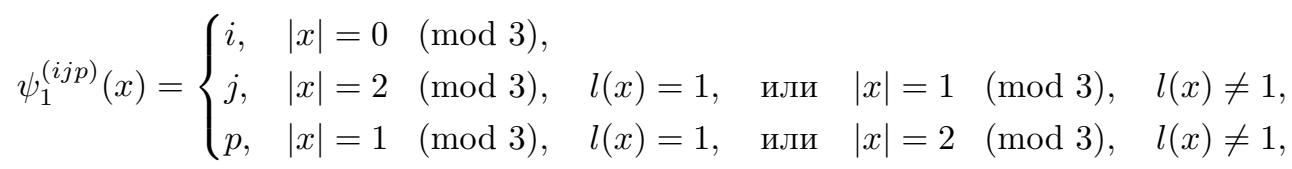

где $i, j, p=1,2,3, i \neq j \neq p$.

Случай $C_{8}$. Для этого класса конфигурации определим

$$
\psi_{2}^{(i j p)}(x)= \begin{cases}i, & r(x)=1, \quad l(x) \neq 3, \quad \text { или } \quad r(x)=3, \quad l(x)=3, \\ j, & r(x)+l(x)=4, \\ p, & r(x)=2, \quad l(x)=1, \quad \text { или } \quad r(x)+l(x)=5, \\ p, & x=e .\end{cases}
$$

Таким образом, мы имеем конфигурацию $\psi_{2}^{(i j p)}$ (вообще говоря, не являющуюся периодической) со свойством $\psi_{2 b}^{(i j p)}=\sigma_{b}$, где $\psi_{2 b}^{(i j p)}-$ сужение конфигурации $\psi_{2}^{(i j p)}$ на $b$. 
Случай $C_{2} \cup C_{5}$. В этом случае конфигурация $\psi_{3}^{(i j p)}$ может быть определена как

$$
\psi_{3}^{(i j p)}(x)=\left\{\begin{array}{lll}
i, & |x|=0 & (\bmod 3) \\
j, & |x|=1 & (\bmod 3) \\
p, & |x|=2 & (\bmod 3)
\end{array}\right.
$$

Случай $C_{5} \cup C_{8}$. В этом случае мы определим конфигурацию $\psi_{4}^{(i j p)}$ следующим образом:

$$
\psi_{4}^{(i j p)}=\left\{\begin{array}{llll}
i, & r(x)=1, \quad l(x) \neq 3, \quad \text { или } \quad r(x)=3, & l(x)=3, \\
j, & r(x)=2, & & \\
p, & r(x)=3, \quad l(x) \neq 3, & \text { или } \quad r(x)=1, & l(x)=3, \\
p, & x=e .
\end{array}\right.
$$

По построению ясно, что для каждой конфигурации

$$
\varphi \in\left\{\varphi^{(i)}, \varphi^{(i j)}, \psi^{(i j)}, \varphi^{(i j p)}, \psi^{(i j p)}, \psi_{1}^{(i j p)}, \psi_{2}^{(i j p)}, \psi_{3}^{(i j p)}, \psi_{4}^{(i j p)}\right\}
$$

мы имеем $\varphi_{b^{\prime}} \in C$, если $\sigma_{b} \in C$ и $C \in\left\{C_{1}, C_{2} \cup C_{6}, C_{3}, C_{9}, C_{5} \cup C_{8} \cup C_{9}, C_{5}, C_{8}, C_{3} \cup\right.$ $\left.C_{5}, C_{5} \cup C_{8}\right\}$ для любого $b^{\prime} \in M$. Теорема доказана.

ЗАмечАниЕ 1. В работе [3] также получены конфигурации $\varphi^{(i)}, \varphi^{(i j)}, \psi^{(i j)}, \varphi^{(i j p)}$, $\psi_{4}^{(i j p)}$ для модели Поттса с конкурирующими взаимодействиями на дереве Кэли. Из классов $C_{4}$ и $C_{7}$ нельзя построить конфигурации. Поэтому эти классы не включены в теорему 1.

Для каждого $m=1,2, \ldots, 9$ обозначим через $A_{m}$ множество точек $J$ таких, что $U_{m}(J)=\min \left(\left\{U_{i}(J)\right\}, i=1,2, \ldots, 9\right)$, т. е. $A_{m}(J)=\left\{J \in \mathbb{R}^{3}: \min \left\{U_{1}(J), U_{2}(J), \ldots\right.\right.$, $\left.\left.U_{9}(J)\right\}=U_{m}\right\}$.

Следующие результаты получены путем непосредственных вычислений:

a) $A_{1}=\left\{J \in \mathbb{R}^{3}: J_{1} \leqslant 0, J_{1}+4 J_{2} \leqslant 0, J_{1}+2 J_{2}+2 J_{3} \leqslant 0\right\}$,

$$
\begin{aligned}
& A_{2}=\left\{J \in \mathbb{R}^{3}: J_{1} \geqslant 0, J_{2} \leqslant 0, J_{2}+J_{3} \leqslant 0\right\}, \\
& A_{3}=\left\{J \in \mathbb{R}^{3}: J_{1} \leqslant 0, J_{1}+4 J_{2} \geqslant 0, J_{2}-J_{3} \geqslant 0, J_{1}+2 J_{3} \leqslant 0\right\}, \\
& A_{4}=\left\{J \in \mathbb{R}^{3}: J_{1}=0, J_{2} \geqslant 0, J_{3} \leqslant 0\right\}, \\
& A_{5}=\left\{J \in \mathbb{R}^{3}: J_{1} \geqslant 0, J_{2} \geqslant 0, J_{2}-J_{3} \geqslant 0, J_{1}-2 J_{3} \geqslant 0\right\}, \\
& A_{6}=\left\{J \in \mathbb{R}^{3}: J_{1} \leqslant 0, J_{1}+2 J_{2} \leqslant 0, J_{2}-J_{3} \leqslant 0, J_{1}+2 J_{2}+2 J_{3} \geqslant 0\right\}, \\
& A_{7}=\left\{J \in \mathbb{R}^{3}: J_{1}=0, J_{2} \leqslant 0, J_{2}+J_{3} \geqslant 0\right\}, \\
& A_{8}=\left\{J \in \mathbb{R}^{3}: J_{1} \geqslant 0, J_{1}-2 J_{2} \geqslant 0, J_{3} \geqslant\left|J_{3}\right|\right\}, \\
& A_{9}=\left\{J \in \mathbb{R}^{3}:\left|J_{1}\right| \leqslant 2 J_{2},\left|J_{1}\right| \leqslant 2 J_{3}\right\} ;
\end{aligned}
$$

б) $A_{1} \cup A_{2} \cup A_{3} \cup A_{5} \cup A_{6} \cup A_{7} \cup A_{8} \cup A_{9}=\mathbb{R}_{+}^{3}$ ( $\left.J_{3}>0\right)$ (рис. 1$)$;

в) $A_{1} \cup A_{2} \cup A_{3} \cup A_{4} \cup A_{5}=\mathbb{R}_{-}^{3}\left(J_{3} \leqslant 0\right)$ (рис. 2);

г) $A_{1} \cup A_{2} \cup A_{3} \cup A_{4} \cup A_{5} \cup A_{6} \cup A_{7} \cup A_{8} \cup A_{9}=\mathbb{R}^{3}$. 


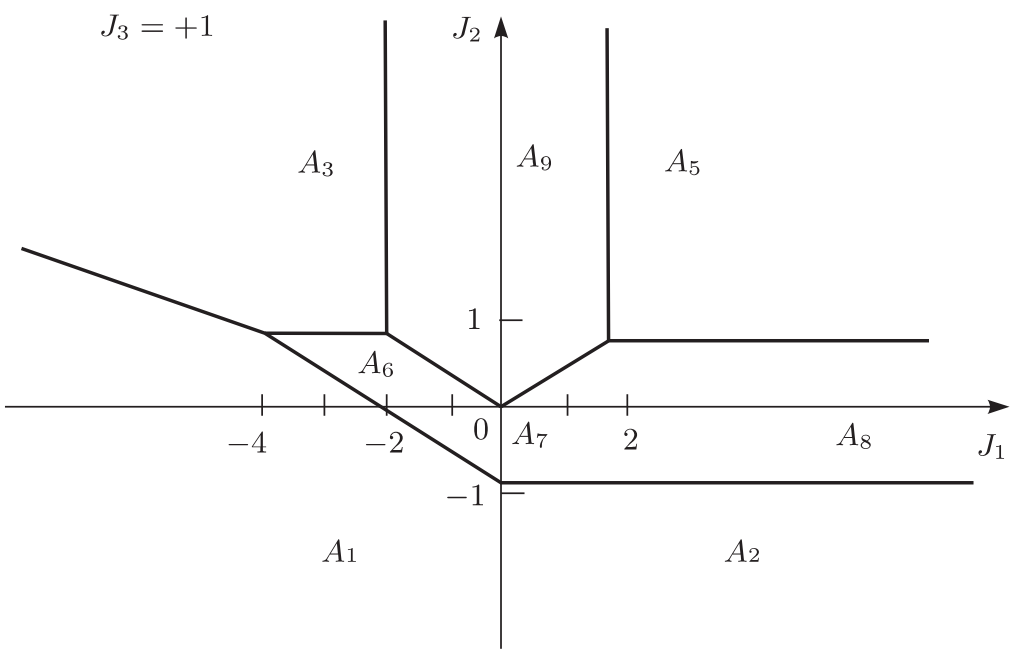

Рис. 1

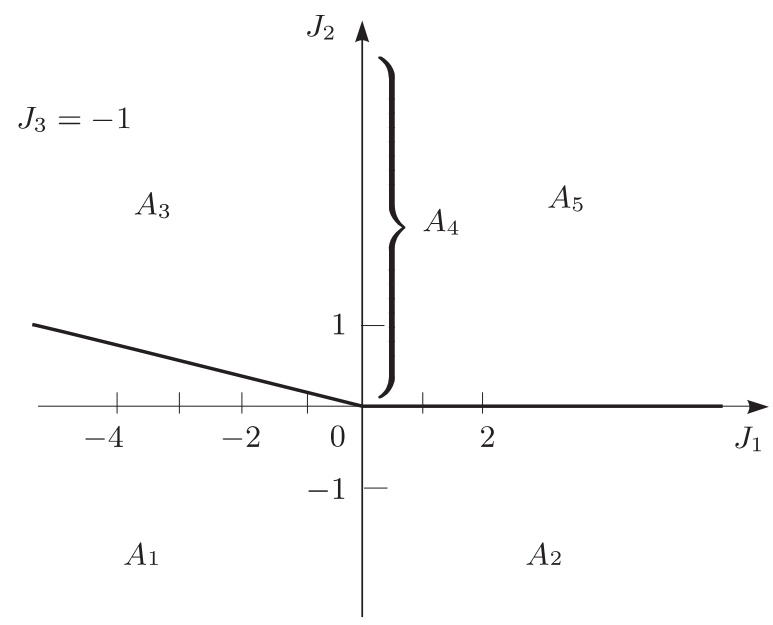

Рис. 2

На рис. 1 и рис. 2 показаны сечения множеств $A_{m}, m=1,2,3,5, \ldots, 9$, плоскостями $J_{3}=a(a>0)$ и сечения множеств $A_{m}, m=\overline{1,5}$, плоскостями $J_{3}=a(a \leqslant 0)$ соответственно.

Введем обозначения:

$$
\begin{aligned}
B_{i_{1} i_{2} \ldots i_{k}} & =A_{i_{1}} \cap A_{i_{2}} \cap \cdots \cap A_{i_{k}}, \quad k=2,3,4, \quad \text { где } \quad 1 \leqslant i_{j} \leqslant 9, \quad j=1, \ldots, k, \\
\widetilde{A_{1}} & =A_{1} \backslash\left(B_{12} \cup B_{13} \cup B_{16}\right), \\
\widetilde{A_{2}} & =B_{36} \backslash\left(B_{369} \cup B_{136}\right), \\
\widetilde{A_{3}} & =A_{2} \backslash\left(B_{12} \cup B_{25} \cup B_{27} \cup B_{28}\right), \\
\widetilde{A_{4}} & =A_{9} \backslash\left(B_{39} \cup B_{49} \cup B_{59} \cup B_{69} \cup B_{89}\right),
\end{aligned}
$$




$$
\begin{aligned}
& \widetilde{A_{5}}=B_{589} \backslash\{(0,0,0)\}, \\
& \widetilde{B_{1}}=A_{5} \backslash\left(B_{58} \cup B_{59} \cup B_{25} \cup B_{35}\right), \\
& \widetilde{B_{2}}=A_{8} \backslash\left(B_{28} \cup B_{58} \cup B_{78} \cup B_{89}\right), \\
& \widetilde{B_{3}}=B_{25} \backslash\left(B_{125} \cup B_{258}\right), \\
& \widetilde{B_{4}}=B_{58} \backslash\left(B_{258} \cup B_{589}\right), \\
& \widetilde{B_{5}}=B_{12} \backslash\left(B_{13} \cup B_{16}\right), \\
& \widetilde{B_{6}}=B_{13} \backslash\left(B_{12} \cup B_{16}\right), \\
& \widetilde{B_{7}}=B_{39} \backslash\left(B_{49} \cup B_{369}\right), \\
& \widetilde{B_{8}}=B_{369} \backslash\{(0,0,0)\} .
\end{aligned}
$$

Здесь $B_{i j}, i, j=\overline{1,9}$, - части плоскостей в $\mathbb{R}^{3}$, а множества $B_{i j k}, i, j, k=\overline{1,9},-$ лучи в $\mathbb{R}^{3}$.

Пусть $G S(H)$ - множество всех основных состояний, а $G S_{p}(H)$ - множество всех периодических основных состояний.

Теорема 2. Справедливы следующие утверждения.

Если $J=(0,0,0)$, тогда $G S(H)=\Omega$.

Если $J \in \widetilde{A_{1}}$, тогда $G S_{p}(H)=\left\{\varphi^{(i)}: i=1,2,3\right\}$.

Если $J \in \widetilde{A_{2}}$, тогда $G S_{p}(H)=\left\{\varphi^{(i j)}: i, j=1,2,3, i \neq j\right\},\left|G S_{p}(H)\right|=3$.

Если $J \in \widetilde{A_{3}}$, тогда $G S_{p}(H)=\left\{\psi^{(i j)}: i, j=1,2,3, i \neq j\right\},\left|G S_{p}(H)\right|=3$.

Если $J \in \widetilde{A_{4}}$, тогда $G S_{p}(H)=\left\{\varphi^{(i j p)}: i, j, p=1,2,3, i \neq j \neq p\right\},\left|G S_{p}(H)\right|=3$.

Если $J \in \widetilde{A_{5}}$, тогда $G S_{p}(H)=\left\{\psi^{(i j p)}: i, j, p=1,2,3, i \neq j \neq p\right\},\left|G S_{p}(H)\right|=6$.

Если $J \in \widetilde{B_{i}}, i=\overline{1,4}$, mогда $G S_{p}(H) \supset\left\{\psi_{k}^{(i j p)}: i, j, p=1,2,3, i \neq j \neq p, k=\overline{1,4}\right\}$.

Здесъ в каждом случае конфигурачии определены в доказательстве теоремы 1.

Если $J \in \widetilde{B_{i}}, i=5,6,7,8$, тогда $\left|G S_{p}(H)\right|=6$.

ДокАзАТЕльство. Первое утверждение тривиально. В остальных случаях для данной конфигурации $\sigma_{b}$, которая делает энергию $U\left(\sigma_{b}\right)$ минимальной, с помощью теоремы 1 можно построить периодические конфигурации $\varphi^{(i j)}, \varphi^{(i j p)}, \psi^{(i j p)}$ (с периодом $p \leqslant 8)$. Для каждого случая точное число таких основных состояний совпадает с числом различных конфигураций $\varphi_{b}$ таких, что энергия $U\left(\varphi_{b}\right)$ будет минимальной для любого $b \in M$. Теорема доказана.

Благодарности. Автор выражает глубокую признательность профессору У. А. Розикову за полезные дискуссии и советы.

\section{Список литературы}

[1] U. A. Rozikov, J. Stat. Phys., 122:2 (2006), 217-235.

[2] U. A. Rozikov, Gibbs Measures on Cayley Trees, World Sci., Singapore, 2013.

[3] Г. И. Ботиров, У. А. Розиков, ТМФ, 153:1 (2007), 86-97.

[4] Н. Н. Ганиходжаев, У. А. Розиков, ТМФ, 111:1 (1997), 109-117.

[5] И. А. Кашапов, ТМФ, 33:1 (1977), 110-118.

[6] П. М. Блехер, Н. Н. Ганиходжаев, ТВП, 35:2 (1990), 220-230. 